\title{
Gamification in Inclusive eLearning
}

\author{
Yasmin Patzer ${ }^{(凶)}$, Nick Russler, and Niels Pinkwart \\ Humboldt-University Berlin, Unter den Linden 6, 10099 Berlin, Germany \\ patzer@informatik.hu-berlin.de \\ https://cses.informatik.hu-berlin.de/en/
}

\begin{abstract}
The usage of gamification elements in learning contexts is getting more and more attention, as it can help increase learners' motivation. Nevertheless accessibility and inclusion are seldom considered yet. In this paper an inclusive gamification concept for an eLearning course is presented. The main target group are people with poor basic education in hospitality industry. The developed gamification concept has been tested in a first small pilot study.
\end{abstract}

Keywords: Gamification · eLearning $\cdot$ Inclusion

The awareness of the importance of inclusion all over society has been increasing over the last years. One big milestone on the way to inclusion is the UN convention on the rights of persons with disabilities. It includes the access to education, no matter what the limitations of a person are. As the importance of eLearning in educational contexts is increasing as well, accessibility and inclusion are requirements that need to be fulfilled here as well. Gamification elements are used increasingly in learning environments to support learner motivation. There are also already some ideas and research on designing for accessible gamification, but not yet in combination with inclusive eLearning. This paper addresses this research gap.

There is a lot of research on inclusion, eLearning and gamification, but not on the combination of all three of them. Inclusive eLearning is a yet rarely researched field with just a few authors' publications [1,2]. Publications on gamification and accessibility are also rare [3]. There are some, which combine those two but rather with a focus on how to use gamification to spread accessibility [4] than on how to make gamification accessible. There are also some publications on how gamification can be used in socio-technical systems that realize different levels of accessibility, such as [5-7]. Yet, as argued, these approaches do not consider the specific requirements of eLearning scenarios.

In cooperation with KOPF, HAND + FUSS and Arbeit und Leben BerlinBrandenburg (DGB/VHS), we designed and developed an eLearning course for people with poor basic education. The course is technically based on the inclusive eLearning platform LAYA [2]. As part of this research, we technically integrated accessible gamification elements in this platform. 
In the course a sequence of videos guides the learner and builds up a realistic story setting in a hotel. Aim of the course is to improve the learners' basic literacy focused on requirements of the hospitality industry. This is addressed by ten different exercises on IT-topics. These exercises are offered on three different levels of difficulty targeting different levels of reading, writing and media literacy skills. To increase the motivation during this learning process gamification elements are included. The gamification concept for this course [8] embeds the learning into a story with characters and tasks and makes the learner the protagonist. Good performance and challenging exercises are rewarded with trophies and badges, which the learner collects throughout the whole course.

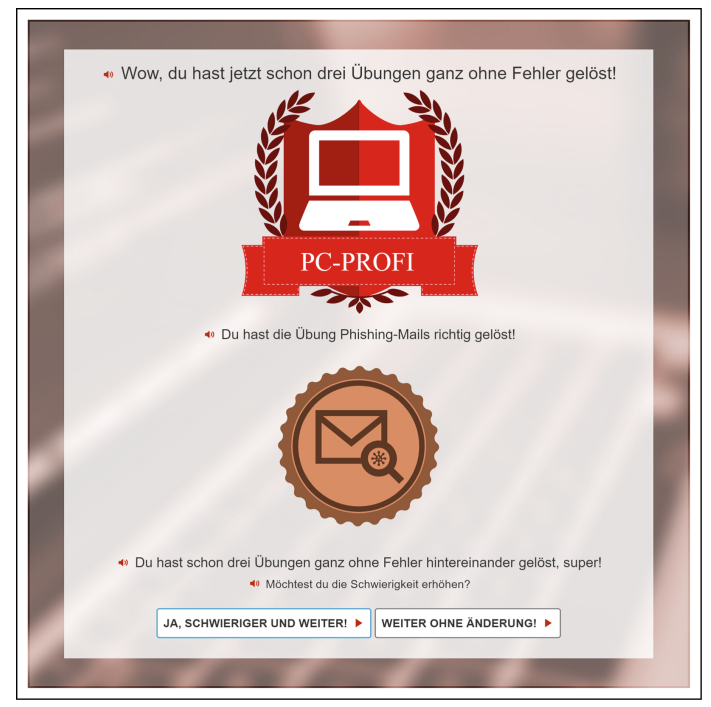

Fig. 1. In-between-page

Following the collection pattern learners collect badges for each solved exercise. The colour of the badges depends on the level of difficulty: bronze for easy, silver for medium and gold for difficult. Related to the exercise content each exercise has an own pictogram shown on its' badges. In Fig. 1 for instance the learner receives a bronze badge for the phishing mail exercise. It is accompanied by the sentence "You have solved the exercise phishing mail correctly". Like the badge colour itself, the text depends on the level of difficulty.

Besides badges learners can collect trophies. The upper part of Fig. 1 shows a trophy with the above text "Wow, you have already solved three exercises without mistakes!" . Trophies are not dependent of single exercises but of solving exercises on the first try. There are three different trophies for one ("PC-Knowledgeable"), two ("Power-User") or three ("PC-Professional") solved exercises without mistakes, with additional complimenting text. The laptop pictogram in the trophy, 
as well as the names of the trophies, is connected to the content of the course as a whole. The trophies themselves are a motivational prize for good performance. Suitable pictograms have been chosen for badges and trophies. To prevent an interruption of the learning process, the text is in easy language and also available as audio. A major requirement was to not create barriers by the in-between-page. Therefore trophies and badges follow the WAI-ARIA recommendations for informative graphics.

Another gamification element, that has been used in this course are recommendations to change the difficulty level of the exercises. These recommendations appear at the bottom of the in-between -page, if the learner solved two exercises without mistakes or was not able to solve two exercises in a row. He is then asked if he wants to increase or decrease the difficulty of the exercises. As illustrated in Fig. 1 the learner has the choice to follow the recommendation or not (and continue on the level that was set before).

After finishing the whole course the learner is presented the completion-page, where all the collected badges and the best received trophy are shown. Beneath each badge the name of the exercise is written, to simplify the relation between badge and exercise for the user. Furthermore, the learner gets congratulations for finishing the course and is additionally complimented for the number of exercises he performed without mistakes. If he did so for more than half the exercises, this is emphasized as well. This design follows the gamification principle "Designing for the Novice, Considering the Elder" [9]. At the bottom of this page is a button for restarting the course, in case learners want to repeat the course to collect badges on a higher difficulty level.

A small pilot study was conducted with nine participants, of whom five had poor basic education and four had no impairments. The focus on participants with poor basic education was chosen, as this is the main target group of the eVideo course. The other four participants were experts on technical development and design and therefore included in this first pilot. All participants were observed as they were using the course for at least $50 \mathrm{~min}$. At the end of this practical sequence the completion-page was opened, although it was not possible to go through the whole course in $50 \mathrm{~min}$. But as the gamification concept should be evaluated, users needed to see this page as well. Afterwards, problem centred guided interviews were conducted to enquire the participant's opinions on the course. They were asked about their usage of digital media and if they already had any experiences with eLearning or gamification. Most of the participants already knew gamification elements from games or learning games. Furthermore, the participants were asked about the different gamification elements in this course and what they liked or disliked about these. Except for one participant with no computer experience, who did not take notice of the trophies and badges during the course, all study participants liked these gamification elements and also the differentiation on difficulty. The recommendations on changing the difficulty were also perceived quite positively. Some users said that the question if they wanted to increase the difficulty made them feel confident. We also asked the participants if they would have liked a form of a social 
competition in the course - e.g. with high scores. Here the opinions varied. Some participants loved this idea and said they would have been even more motivated, while others stated that they were learning for their own progress and thus would not want to be part of a competition.

The results of the lessons learned during system development and of the small-scale pilot evaluation can be summarized as follows:

1. The adaptation of the included gamification elements and mechanisms has not been perceived as a barrier. It has either been perceived as motivating or was ignored.

2. The offer to adapt the difficulty level, based on an automatic assessment of the learner's performance, can be meaningful, motivating and effective in an inclusive eLearning system.

3. As impairments often come along with poor media skills, precognition of gamification principles cannot be assumed.

4. Independent of impairments or previous knowledge, gamification in form of social competition might be motivational, but should be optional.

Further studies with a more heterogeneous user bases are necessary to make sure that the course is as accessible and inclusive as possible. A question for further research could be if the positive perception of the tested gamification patterns differs for people with other impairments and needs. And if it does, what adaptations are necessary to make the gamification elements more inclusive?

\section{References}

1. Draffan, E.A., Wald, M., Dickens, K., Zimmermann, G., Kelle, S., Miesenberger, K., Petz, A.: Stepwise approach to accessible MOOC development. Stud. Health Technol. Informat. 217, 227 (2015)

2. Patzer, Y., Pinkwart, N.: Inclusive e-learning - towards an integrated system design. In: Cudd, P., De Witte, L. (eds.) Studies in Health Technology and Informatics, vol. 24. IOS Press, Amsterdam (2017)

3. Johnson, D., Deterding, S., Kuhn, K.A., Staneva, A., Stoyanov, S., Hides, L.: Gamification for health and wellbeing: a systematic review of the literature. Internet Interv. 6, 89-106 (2016)

4. Spyridonis, F., Daylamani-Zad, D., Paraskevopoulos, I.T.: The gamification of accessibility design: A proposed framework. In: 2017 9th International Conference on Virtual Worlds and Games for Serious Applications (vs-games), pp. 233-236. IEEE (2017)

5. Korn, O., Muschick, P., Schmidt, A.: Gamification of production? A study on the acceptance of gamified work processes in the automotive industry. In: Chung, W., Shin, C.S. (eds.) Advances in Affective and Pleasurable Design: Proceedings of the ahfe 2016 International Conference on Affective and Pleasurable Design, pp. 433445. Springer International Publishing, Cham (2017). https://doi.org/10.1007/9783-319-41661-8_42

6. Seaborn, K., Edey, J., Dolinar, G., Whitfield, M., Gardner, P., Branje, C., Fels, D.I.: Accessible play in everyday spaces: mixed reality gaming for adult powered chair users. ACM Trans. Comput. Hum. Interact. (TOCHI) 23(2), 12 (2016) 
7. Jent, S., Janneck, M.: Gamification für blinde und sehbehinderte Menschen. In: Burghardt, M., Wimmer, R., Wolff, C.,Womser-Hacker, C. (eds.) Mensch und Computer 2017 - Tagungsband, pp. 341-344. Regensburg: Gesellschaft für Informatik e.V (2017)

8. Russler, N.: Gamification in inklusivem E-Learning. Master Thesis. HumboldtUniversity Berlin, Department of Informatics (2018)

9. Zichermann, G., Cunningham, C.: Gamification by Design: Implementing Game Mechanics in Web and Mobile Apps. O'Reilly Media Inc., Sebastopol (2011)

Open Access This chapter is licensed under the terms of the Creative Commons Attribution 4.0 International License (http://creativecommons.org/licenses/by/4.0/), which permits use, sharing, adaptation, distribution and reproduction in any medium or format, as long as you give appropriate credit to the original author(s) and the source, provide a link to the Creative Commons license and indicate if changes were made.

The images or other third party material in this chapter are included in the chapter's Creative Commons license, unless indicated otherwise in a credit line to the material. If material is not included in the chapter's Creative Commons license and your intended use is not permitted by statutory regulation or exceeds the permitted use, you will need to obtain permission directly from the copyright holder.

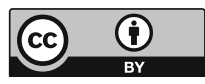

